\title{
A model for airline seat control considering risk and discount
}

\author{
Wei Fan \\ Computer Institute, Civil Aviation university of \\ China,Tianjin China. \\ wfancauc@163.com
}

\author{
Jianwei Wang \\ Computer Institute, Civil Aviation university of China, \\ Tianjin China. \\ 252588389@qq.com
}

\begin{abstract}
Most seat control models rely on the concept of marginal seat revenue and the assumption of risk neutrality to develop an policy to solve this kind of problem. For the multi-leg dynamic seat control problem ,this study proposed a modified multi-leg policy to take risk into consideration by discounting the marginal seat revenue and using the virtual bucket .The effectiveness of the modified policy was examined by a series of simulation experiments. The simulation analysis shows that the model can balance the expected revenue and revenue variation.
\end{abstract}

Keywords-seat control models, riskneutrality, multi-leg, virtualbucket, simulation.

\section{INTRODUCTION}

The airline seats optimization means selling the appropriate seat to the right person at the right time.If the airline seats are all sold at the cheap price. Therefore, the purpose of optimization of airline seats is allocated reasonable ticket protection seat numbers for the different levels of fares so as to gain the maximization of the flight revenue.

Today airline seatsoptimal control method is mainly theexpected marginal seat revenue( EMSR) theory which proposed by the Professor Peter P.Belobaba in his doctoral thesis. This theory was quickly adopted by many airline company including PROS and other multiple software systems. But EMSR has two disadvantages, First, a reservation limit that EMSR produced cannot change as thereservation process state change, which called the drawback of static characteristics. The second is not considered the risk in the decision-making process. For these two drawbacks, foreign countries has proposed the discount of expected marginal revenuethinking, but this method is applied only in the single-leg. For the multi-leg problem has not a solution to this. Recent years the multi-leg problem's solution is to use the virtual bucket, sothis study propose a model using the virtual bucket and the discount thinking to solve this problem.

\section{BACKGROUND AND PRIOR RESEARCH}

\section{A. Original Discounted Marginal Seat Value}

One of the policies which Huang and Chang (2009) propose is the relaxation of optimalityfor a more risk-sensitive policy. They show in a numerical experiment the behavior of thispolicy in terms of average and standard deviation. This policy discounts the marginal seatvalue. Its value function is

$$
\begin{gathered}
\mathrm{V}_{n}^{\beta}= \\
\left\{\begin{array}{c}
r_{i}+\sum_{i=0}^{k} p_{i n} V_{n-1}^{\beta}(c-1, i) r_{i} \gg \beta \cdot \delta_{V_{n-1}^{\beta}}(c, i) \\
\sum_{i=0}^{k} p_{i n} V_{n-1}^{\beta}(c-1, i) \\
\text { oisa discount factor which ranged in }[0, \ldots, 1] \text { And the }
\end{array}\right. \\
\text { decision rules is } \\
f_{n}^{\beta}(c, i)=\left\{\begin{array}{l}
1, r_{i} \gg \beta \cdot \delta_{V_{n-1}^{\beta}}(c, i) \\
0, r_{i}<\beta \cdot \delta_{V_{n-1}^{\beta}}^{\beta}(c, i)
\end{array}\right) \\
\text { B. Discounted Marginal Seat Value within Risk-Neutral } \\
\text { Solution }
\end{gathered}
$$

\section{B. Discounted Marginal Seat Value within Risk-Neutral Solution}

The idea in 2.1 using the marginal seat value is based on previous discount calculation result which will decrease the value largely in several accumulations. So this solution work directly with the marginal seatvalue with a prior computed risk-neutral value function. Thus, the decisionrules do not depend on previous 'discounted' decisions.

The modified decision rules is as followed,

$$
f_{n}^{\beta, \mathrm{v}}(c, i)=\left\{\begin{array}{l}
1, r_{i} \gg \beta \cdot \delta_{V_{n-1}^{*}}(c, i) \\
0, r_{i}<\beta \cdot \delta_{V_{n-1}^{*}}(c, i)
\end{array}(3)\right.
$$

$\delta_{V_{n-1}^{*}}(c, i)$ Is the marginal seat value within risk-neutral solution at stage $n-1$ when the residual seat is $c$ and required class level is $i$.The value function $\mathrm{V}_{n}^{\beta}$ would be transformed similarly toobtain $\mathrm{V}_{n}^{\beta, \mathrm{v}}$.

\section{Selling-Rate Dependent Decisions}

The discount $\beta$ in different stage should be a different value, so the model before is not a realistic one. So this solution is the time- and seat-dependent compromise policy of Huang and Chang(2009). It uses a hyperbolic tangent function and two variable parameter $k_{1}$ and $k_{2}$ whichdetermine the level of risk-sensitive behavior in dependence on the number of remainingseats before departure. The discount factor $\beta_{n}^{k_{1}, k_{2}}(c)$ is computed as

$$
\begin{aligned}
& \beta_{n}^{k_{1}, k_{2}}(c)=\frac{1}{2}\left[\tanh \left(k_{1}\left(q(n)+k_{2}-c\right)\right)+1\right](4) \\
& \tanh (x)=\frac{e^{x}-e^{-x}}{e^{x}+e^{-x}}(5) \\
& \mathrm{q}(n)=C \frac{\sum_{m=1}^{n} \sum_{i=1}^{k} r_{i} p_{i n}}{\sum_{m=1}^{N} \sum_{i=1}^{k} r_{i} p_{i n}}(6) \\
& \text { - } \quad k_{1}: \text { a pre-determined constant representing the }
\end{aligned}
$$
rate of decision change. $k_{1}$ Was chosen as 0.3 .

- $\quad k_{2}$ :An adjustable parameter representing the level of risk-aversion in range[2,5]. 


\section{MODEL AND POLICIES DESCRIPTION}

\section{A. model description}

This studyusesMarkov decision processes as a dynamic decision-making booking process.

A Markovdecision-making process, includingthestate space, action set, decisionepochs, rewards and transition probabilities.Inthestudyherein, which are defined as follows:

- State space $S=\{0,1 \ldots C\} \times\{0,1 \ldots \mathrm{k}\}$, wherethe first element stands for the remaining seat capacity and the second element for the fare class, with artificialfare class 0 with fare $\mathrm{F} 0=0$. A state $(\mathrm{c}, \mathrm{i})$ says that as $\mathrm{c}$ seats are remaining, we have a requestfor fareclass $i$.

- Action set:A $(c, \mathrm{i})=\{0,1\}, \forall(c, \mathrm{i}) \in S \mid \mathrm{i}>0, A(c, 0)=$ $\{0\}$, Represents the 'reject' and 'accept' decision for a given state.

- Decision epochs correspond to the time periods: $\mathrm{T}=\{0,1, . . \mathrm{N}\}, \mathrm{N}$ represents the departure of flight, 0 is the start of booking.

- transition probabilities: $q_{n}((c, \mathrm{i}), \mathrm{a},(c-\mathrm{a}, \mathrm{j}))=p_{j n}$

- Rewards: $r_{n}((c, \mathrm{i}), \mathrm{a})=\mathrm{a} \cdot r_{i}, \mathrm{c}>0$

Combined discount idea and virtual bucket, first we should divide the bucket according to the multi-leg fares, and then put the bucket to the corresponding leg. Eventually we calculate the expected marginal seat value at every stage according to one of three method above.

The protection number function is as follows:

$$
y_{i-1}^{* \beta}=\max \left\{r_{i}<\sum_{i=0}^{k} p_{i n}\left(V_{n-1}^{\beta}(c, i)-V_{n-1}^{\beta}(c-\right.\right.
$$

1,i))\} $\mathrm{c} \in N_{0}(7)$

\section{B. The division of stage and the transition probabilities' setting}

- The booking time is divided into a series of short time, andin each time intervalthe passenger arrival process follows a Poisson distribution.

- Each interval was set a number $n, n=1,2,3 \ldots h$. Timeintervallon behalf of the end of booking time,time intervalhon behalf of the beginning of booking.

- Since the model assumes that each decision interval at most only one tourist arrivals,so each time interval described above is divided into intervals of the decisions required by the model. $\mu_{\mathrm{n}}^{\mathrm{k}}$ Means the expected arrivals in stage $\mathrm{n}$ required class level k.Each class level's expected arrivals in every stage satisfies the following formula: $\mu^{\mathrm{n}}=$ $\mu_{1}^{\mathrm{n}}+\mu_{2}^{\mathrm{n}}+\cdots+\mu_{\mathrm{k}}^{\mathrm{n}}$, Each time interval travelers arrive meet the Poisson distribution with mean $\mu^{\mathrm{n}}$.

- The interval $\mathrm{n}$ is divided into $v^{n}$ decision short interval of equal length.Passengers arrival in each short interval meet the Poisson distribution with mean $\mu^{n} / v^{n}$, and the most important part is each interval at most arrive one passenger.

- $\varepsilon$ is a very small probability value which can be negligible, thenv ${ }^{\mathrm{n}}$ must satisfy the formulaP $(x \gg 2)<\varepsilon$, where $\mathrm{x}$ is a random number, representing thenumber of passengersarriving withina decisioninterval.Because each interval meet the Poisson distribution with mean $\mu^{\mathrm{n}} /{ }_{\mathrm{v}}$, then $\mathrm{v}^{\mathrm{n}}$

satisfy: $1-\exp \left(-\frac{\mu^{n}}{v^{n}}\right)-\left(\mu^{n} / v^{n}\right) \exp \left(\mu^{n} / v^{n}\right) \ll \varepsilon$

- Calculate the passenger arrival's probability of each decision interval. Because passengers arrival meet the Poisson distribution withmean $\mu^{\mathrm{n}} / \mathrm{v}^{\mathrm{n}}, \mathrm{P}_{\mathrm{k}}(\mathrm{n})=\left(\mu^{\mathrm{n}} / \mathrm{v}^{\mathrm{n}}\right) \exp \left(-\mu^{\mathrm{n}} / \mathrm{v}^{\mathrm{n}}\right), \mathrm{P}_{\mathrm{k}}(\mathrm{n})$ means a passenger arrive requiring class level $\mathrm{k}$ at stage $\mathrm{n}$.

\section{NUMERICAL SIMULATION AND RESUlTS}

This study uses the domestic airline Nanjing (A) --Shenzhen (B) --- Sanya (C) routes data to do the simulation. Each ODF has two class level fares, The aircraft available seat number is 140 , and there are three segments, $A B$, $\mathrm{AC}, \mathrm{BC}$.ODF fares and demand information is as shown in Table 1.

\begin{tabular}{ccccccc}
\multicolumn{7}{c}{ Table 1 ODF fares and demand information } \\
\hline ODF & ABY & ABB & ACY & ACB & BCY & BCB \\
\hline fare & 1000 & 800 & 1200 & 960 & 400 & 320 \\
demand & 42 & 66 & 35 & 50 & 41 & 71 \\
\hline
\end{tabular}

Because the ODF is not too much, so we take each fare as a bucket.Then there are 6 virtual buckets. For experimental data,the results of bucket division are as follows in Table 2:

Table 2 the result of bucket division

\begin{tabular}{llllll}
\hline AB bucket & ODF class & fare & BC bucket & ODF class & fare \\
\hline 1 & ACY & 1200 & 1 & ACY & 1200 \\
2 & ABY & 1000 & 2 & ACB & 960 \\
3 & ACB & 960 & 3 & BCY & 400 \\
4 & ABB & 800 & 4 & BCB & 320 \\
\hline
\end{tabular}

Because passengers arrival meet nonhomogeneous Poisson distribution, so we can get the probabilityand the number of passengers arrival at each stage according to the section 2.3.In our case, the stimulation result are as follows: Table 3 Probabilities of passengers arrival

\begin{tabular}{lllllll}
\hline stage & ACY & ABY & ACB & ABB & BCY & BCB \\
\hline $0-180$ & 0.037 & 0.042 & 0.011 & 0.016 & 0.027 & 0.011 \\
$181-360$ & 0.027 & 0.032 & 0.016 & 0.027 & 0.027 & 0.022 \\
$361-510$ & 0.025 & 0.032 & 0.019 & 0.019 & 0.032 & 0.026 \\
$511-660$ & 0.019 & 0.025 & 0.019 & 0.025 & 0.025 & 0.025 \\
$661-840$ & 0.016 & 0.021 & 0.021 & 0.027 & 0.021 & 0.032 \\
$841-990$ & 0.019 & 0.019 & 0.025 & 0.032 & 0.025 & 0.038 \\
$991-1170$ & 0.016 & 0.016 & 0.027 & 0.037 & 0.016 & 0.042 \\
$1171-1350$ & 0.010 & 0.016 & 0.032 & 0.037 & 0.016 & 0.042 \\
$1351-1550$ & 0.010 & 0.010 & 0.029 & 0.038 & 0.014 & 0.043 \\
$1551-1750$ & 0.010 & 0.010 & 0.029 & 0.043 & 0.014 & 0.047 \\
$1751-1950$ & 0.005 & 0.010 & 0.038 & 0.047 & 0.019 & 0.010 \\
\hline \multicolumn{7}{c}{ Table 4 the situation of Passengers arrival }
\end{tabular}

ODF It has a arrival at interval $\mathrm{t}$

ABY 48,55,67,71,84,93,173,200,208,217,232,247,260,262,333,398,413, $435,444,461,504,521,533,544,547,560,594,642,661,703,783,869$, $999,1004,1071,1103,1120,1163,1217,1221,1276,1302,1322,1383$, $1438,1536,1618,1693,1737,1763,1828,1919$

ABB $79,148,177,223,228,258,266,314,354,411,428,448,483,484,498,577$, $628,709,712,732,756,789,817,833,853,862,868,935,991,1019,1106$, $1187,1193,1219,1230,1317,1319,1335,1336,1401,1409,1424,1483$, $1492,1506,1520,1537,1538,1647,1673,1677,1684,1710,1747,1782$, $1788,1806,1840,1842,1846,1853,1866,1867,1893,1905,1914,1942$ 


\begin{tabular}{ll}
\hline ACY & $11,30,31,46,74,89,90,96,127,214,219,246,265,337,359,409,447,477$, \\
& $489,492,526,530,561,602,607,608,635,648,689,701,832,854,889,917$, \\
& $925,933,939,982,1026,1107,1207,1471,1597,1751,1845$ \\
ACB & $116,321,433,456,497,571,600,625,636,691,696,720,729,791,830,846$, \\
& $888,988,1042,1052,1129,1226,1255,1282,1315,1329,1330,1368,1388$, \\
& $1407,1488,1531,1535,1727,1739,1755,1767,1778,1834,1874,1878$, \\
& $1922,1923,1936,1943$ \\
BCY & $17,24,65,92,215,251,285,295,303,341,345,367,368,387,422,424,458$, \\
& $462,469,488,494,511,546,651,656,700,704,719,731,735,823,855$, \\
& $914,920,937,951,973,983,1032,1047,1114,1120,1229,1235,1236$, \\
& $1269,1289,1405,1616,1654$ \\
BCB & $18,292,334,374,382,468,474,524,615,641,713,761,812,816,842,852$, \\
& $865,877,885,913,964,974,1003,1025,1046,1049,1059,1118,1125$, \\
& $1132,1160,1180,1184,1207,1234,1266,1311,1341,1342,1343,1380$, \\
& $1394,1399,1454,1470,1509,1512,1517,1519,1530,1560,1623,1682$, \\
& $1697,1713,1731,1775,1799,1813,1823,1850,1856,1865,1882,1891$, \\
& $1902,1912,1924,1932,1934,1945$
\end{tabular}

A. Original Discounted Marginal Seat Value simulation

The discount factor $\beta$ was set from 0.8 to 0.9 which was a reasonable arrangement. First we set $\beta=0.9$.

Segment AB's and BC's protection level is as shown:

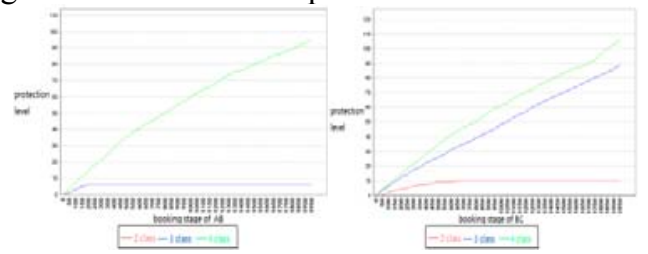

Fig 1:Segment AB's and BC's protection level

We changed the factor $\beta$ to 0.8 ,so we can get the different result as follows:

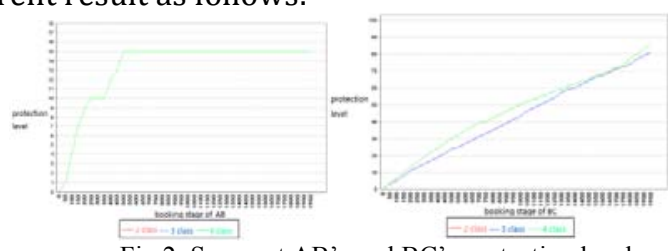

Fig 2: Segment AB's and BC's protection level

The contrast between 0.8 and 0.9 ,we get that when the discount is low, the decision-making strategies approximates FCFS.

\section{B. Discounted Marginal Seat Value within Risk-Neutral Solution simulation}
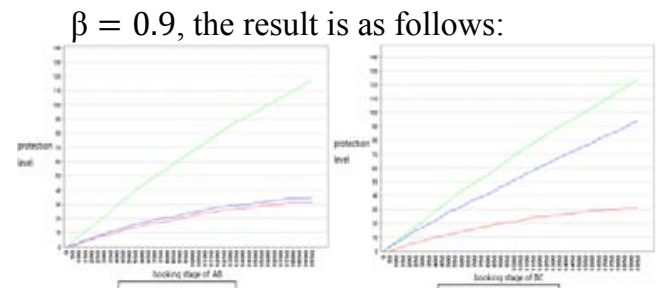

Fig3: Segment AB's and BC's protection level $\beta=0.8$, the result is as follows:

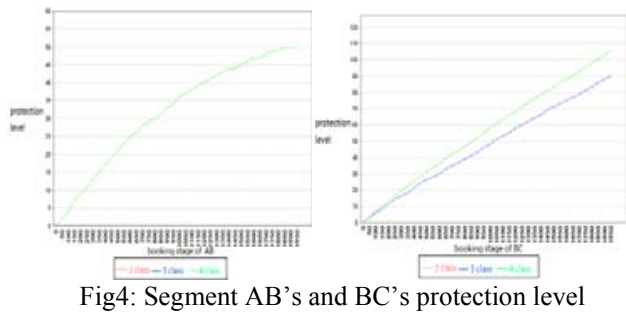

C. Selling-Rate Dependent Decisions simulation

In this model we set $k_{1}=0.3, k_{2}=5$, the simulation of segment $\mathrm{AB}, \mathrm{BC}$ is as follows:

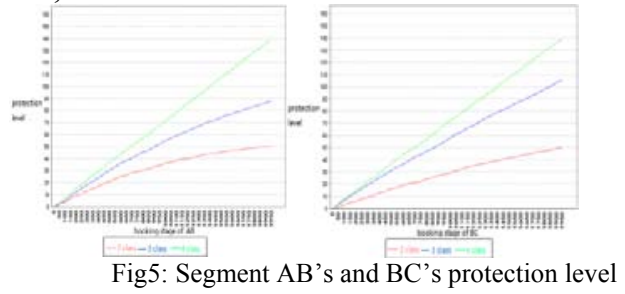

\section{Contrast among three methods}

We named this three methods orderly as method 1 , method 2, method 3, the result of contrast is shown :

\begin{tabular}{llllllll}
\hline Method $(\boldsymbol{\beta})$ & ABY & ABB & ACY & ACB & BCY & BCB & revenu \\
\hline Method1(0.8) & 27 & 50 & 23 & 40 & 8 & 37 & 149240 \\
Method1(0.9) & 37 & 32 & 30 & 41 & 8 & 36 & 152680 \\
Method2(0.8) & 31 & 44 & 25 & 40 & 8 & 36 & 149320 \\
Method2(0.9) & 36 & 22 & 40 & 42 & 8 & 26 & 153440 \\
Method3 & 39 & 18 & 42 & 41 & 6 & 14 & 152040 \\
\hline
\end{tabular}

From this result we can find the method $2(\boldsymbol{\beta}=\mathbf{0 . 9})$ is the best, but the booking result is depend on the passengers' arrival situation. When the situation of arrival is changed, the total revenue will change too. So we can't tell which risk-aversion method is the best, but we can get which one is realistic. This study's purpose is to propose a strategy and give a result of allocation which result will change as the arrival situation changed.

\section{CONCLUSION}

This paper first combined the discount thinking and the virtual bucket using the Markov decision process to solve the multi-leg seat allocation problem. And using the Non-homogeneous Poisson distribution we stimulate the passengers'arrival. We experiment this three methods, we are not to illustrate which method is good, but in order to provide a strategy of this case. Because of taking the risk into this by discount the expected marginal seat value, we break the constraint of purely rational decision, which is more realistic.

\section{REFERENCES}

[1] B.C.Smith,J.F.Leimkuler, and R.M.Darrow.Yield management at American airlines[J].Interfaces,vol.22.no.1,1992.

[2] Matthias Koenig, JoernMeissner. Risk Minimizing Strategies for Revenue Management Problems with Target Values [J]. eprints.lancs.ac.uk,2009. 
[3] Huang, K. and Chang, K. (2009). A simulation analysis for the airline seat control considering expected revenue and risk. Journal of Revenue and Pricing Management

[4] Weatherford LR.EMSR versus EMSU: revenue or utility? [J],Revenue Pricing Manage 3(3):277-284,2004.

[5] Joongwoo Brian Park. Capacity control in network revenue management: Clustering and risk-aversion. USA: MIT, 2010.

[6] C. Barz,K.-H.Waldmann.Risk-sensitive capacity control in revenue management[J], Springer-Verlag,2006. 